\title{
Crystal and magnetic structure of $\mathrm{CeVO}_{3}$
}

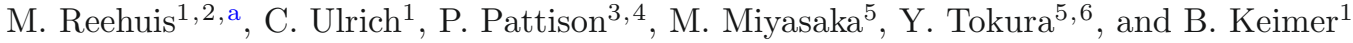 \\ 1 Max-Planck-Institut für Festkörperforschung, 70569 Stuttgart, Germany \\ 2 Hahn-Meitner-Institut, Glienicker Strasse 100, 14109 Berlin, Germany \\ 3 SNBL at ESRF, BP 220, 38042 Grenoble Cedex 9, France \\ 4 Laboratory of Crystallography, Swiss Federal Institute of Technology, BSP-Dorigny, 1015 Lausanne, Switzerland \\ 5 Department of Applied Physics, University of Tokyo, 113 Tokyo, Japan \\ ${ }^{6}$ Correlated Electron Research Center (CERC), National Institute of Advanced Industrial Science and Technology (AIST), \\ Tsukuba 305-8562, Japan
}

Received 13 September 2007 / Received in final form 23 April 2008

Published online 16 July 2008 - (C) EDP Sciences, Società Italiana di Fisica, Springer-Verlag 2008

\begin{abstract}
The crystal structure and the magnetic ordering pattern of the electrically insulating perovskite $\mathrm{CeVO}_{3}$ was investigated by high-resolution powder X-ray diffraction and single-crystal neutron diffraction. A structural phase transition from an orthorhombic to a monoclinic structure (with space groups $\mathrm{Pbnm}$ and $P 2_{1} / b$, respectively) was observed upon cooling below $T_{\mathrm{S}}=136 \mathrm{~K}$. This transition is associated with a strong distortion of the $\mathrm{VO}_{6}$-octahedra and can be attributed to orbital ordering. A magnetic ordering transition driven by exchange interactions between vanadium moments is observed at $T_{\mathrm{N}}=124 \mathrm{~K}$, and antiferromagnetic interactions between magnetic moments on vanadium and cerium ions induce a progressive magnetic polarization of the cerium sublattice at lower temperatures. The full magnetic structure is described by a superposition of the modes $\left(C_{x}, F_{y},-\right)$ and $\left(F_{x}, C_{y},-\right)$. The unit cell volume and the tilt angles of the $\mathrm{VO}_{6}$-octahedra in the $\mathrm{CeVO}_{3}$-crystal structure are anomalous compared to those of other members of the series $R \mathrm{VO}_{3}$ ( $R=$ lanthanide atom), and the ordered magnetic moments on both vanadium and cerium sublattices at low temperatures are considerably smaller than the free-ion values of $\mathrm{V}^{3+}$ and $\mathrm{Ce}^{3+}$. Possible origins of this behavior are discussed.
\end{abstract}

PACS. $61.10 . \mathrm{Nz}$ X-ray diffraction - 61.12.Ld Neutron diffraction - 61.50.Ks Crystallographic aspects of phase transformations; pressure effects $-75.25 .+\mathrm{z}$ Spin arrangements in magnetically ordered materials

\section{Introduction}

Ternary transition-metal oxides with perovskite structure have attracted considerable attention due to their unusual phase behavior and physical properties, which are consequences of an intricate ineterplay between charge, orbital, and spin ordering. Orthovanadates of chemical composition $R \mathrm{VO}_{3}$, with $R=\mathrm{Y}$ or a trivalent rare-earth metal atom, are particularly interesting examples, as they undergo multiple orbital and magnetic ordering transitions as a function of temperature [1-8]. At room temperature these materials exhibit the orthorhombic $\mathrm{GdFeO}_{3}$-type structure with space group Pbnm [9-11]. With decreasing temperature, they undergo a structural phase transition, ascribed to orbital ordering, into a phase described by the monoclinic space group $P 2_{1} / b$ or its sub-groups, and a Néel transition associated with the formation of a $C$-type magnetic structure of the spin- 1 moments of $\mathrm{V}^{3+}$. For some members of this family, including in particular the extensively investigated $\mathrm{YVO}_{3}$, a second structural tran-

\footnotetext{
a e-mail: reehuis@hmi.de
}

sition back to the orthorhombic space group Pbnm and a rearrangement of the magnetic moments into a $G$-type structure are observed at lower temperatures. Finally, ordering of the magnetic moments of the rare-earth ions $R^{3+}$ (if present) occurs at the lowest temperatures.

$\mathrm{CeVO}_{3}$ plays a special role in the family $\mathrm{RVO}_{3}$. In line with other members of the series, this compound exhibits two phase transitions below room temperature, which are attributed to orbital and magnetic ordering. However, both the transition temperatures and the sequence in which orbital and magnetic order is formed have been disputed. While Ren et al. [12] reported structural and magnetic transitions at $T_{\mathrm{S}}=154 \mathrm{~K}$ and $T_{\mathrm{N}}=134 \mathrm{~K}$, respectively, the opposite sequence was reported by Miyasaka et al. [4] $\left(T_{\mathrm{N}}=133 \mathrm{~K}, T_{\mathrm{S}}=122 \mathrm{~K}\right)$ and Muñoz et al. [13] $\left(T_{\mathrm{N}}=136 \mathrm{~K}, T_{\mathrm{S}}=124 \mathrm{~K}\right)$. Moreover, both transition temperatures are anomalously low and the unit cell volume is anomalously small compared to those of other members of the series $[4,7,14]$. Since cerium is found not only in the typical trivalent state, but also in tetravalent state or intermediate-valent states, a possible origin of the 
anomalous properties of $\mathrm{CeVO}_{3}$ is a deviation of the cerium valence from the usual $R^{3+}$. Charge neutrality would then imply an anomalous vanadium valence as well. It is thus interesting to explore the structural and magnetic properties of $\mathrm{CeVO}_{3}$ and their relationship to the Ce and $\mathrm{V}$ valence states in more detail. A recent study including neutron diffraction, specific heat, and magnetization measurements on polycrystalline $\mathrm{CeVO}_{3}$ has addressed this issue [13]. However, the results of this study show many inconsistencies with data reported in the prior literature $[12,15]$. In the present work we have therefore carried out a comprehensive reinvestigation of the structural and magnetic structure of $\mathrm{CeVO}_{3}$ by synchrotron X-ray powder diffraction and single-crystal neutron diffraction.

The paper is organized as follows. Section 2 provides details of the X-ray and neutron diffraction experiments. In Section 3 we first address the sequence of structural and magnetic phase transitions, before describing the crystal structure and magnetic structure of $\mathrm{CeVO}_{3}$ in detail. Section 4 contains conclusions.

\section{Experimental details}

The crystal and magnetic structure as well as the thermal variation of Bragg reflections of $\mathrm{CeVO}_{3}$ have been investigated by single-crystal neutron diffraction. Data sets were collected on the four-circle diffractometer E5 at the BER II reactor of the Hahn-Meitner-Institut in Berlin, Germany. A pyrolytic-graphite monochromator was used to select the neutron wavelength $\lambda=2.36 \AA$. For the experiment we used an untwinned cylindrical single crystal of $\mathrm{CeVO}_{3}$ with dimensions $d=3 \mathrm{~mm}$ and $h=3 \mathrm{~mm}$. The crystal was grown by a floating-zone method as described elsewhere [16]. Data reduction and crystal structure refinements were carried out with the program Xtal 3.4 [17]. For the absorption correction the program ABSORB in the Xtal system takes any shape of the crystal into account. Using the absorption coefficient $\mu=0.562 \mathrm{~cm}^{-1}$ the absorption-weighted individual mean path length through the sample has been determined for every reflection. The individual path lengths were used for the extinction correction, where we applied the method of Zachariasen (Gaussian mosaic-spread distribution). For the refinement of the crystal structure we have used the nuclear scattering lengths $b(\mathrm{O})=5.805 \mathrm{fm}, b(\mathrm{~V})=-0.3824 \mathrm{fm}$, and $b(\mathrm{Ce})$ $=4.84 \mathrm{fm}[18]$. The magnetic moments of the V-and Ceatoms were refined with the program FullProf [19]. The magnetic form factors of the metal ions were taken from reference [20].

In order to investigate in detail the orthorhombic and monoclinic structure of $\mathrm{CeVO}_{3}$ high-resolution powder diffraction studies were performed at the European Synchrotron Radiation Facility in Grenoble. For the experiment we took a single crystal sample, and ground a fragment to powder, which was mounted in a glass capillary of diameter $0.3 \mathrm{~mm}$. Complete powder patterns on the BM1B (Swiss-Norwegian) beam line with a $2 \theta$-range between 1 and $55^{\circ}$ were collected using the wavelength $\lambda=0.5001 \AA$. The uncertainty of the wavelength is less

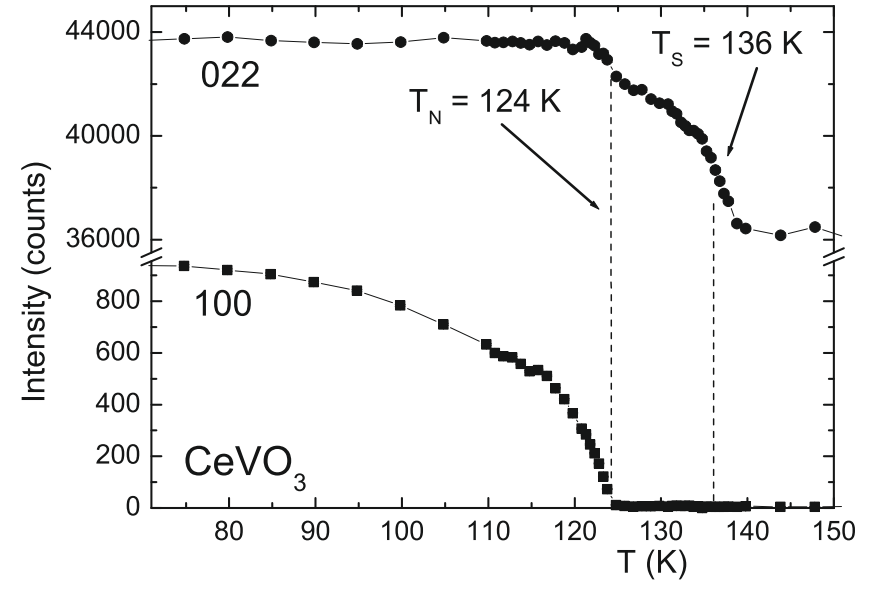

Fig. 1. Temperature dependence of the nuclear and magnetic reflections 022 and 100 of $\mathrm{CeVO}_{3}$. The anomaly at $136 \mathrm{~K}$ observed for the 022 indicates the structural phase transition from the orthorhombic $(P b n m)$ into the monoclinic structure $\left(P 2_{1} / b\right)$. The second anomaly of the 022 as well as the spontaneous increase of the 100 at $124 \mathrm{~K}$ shows the magnetic phase transition where the $C$-type ordering of the vanadium sublattice sets in.

than \pm 0.001 . Rietveld refinements of the powder diffraction data were carried out with the program FullProf using the atomic scattering factors provided by the program [19].

\section{Results and discussion}

\subsection{Sequence of phase transitions}

Before discussing the crystal structure and the magnetic structure of $\mathrm{CeVO}_{3}$, we first address the sequence of structural and magnetic phase transitions, which has been disputed in the literature. To this end, we have measured on E5 the temperature dependence of particular Bragg reflections characteristic of both types of order. The strongest magnetic intensity was observed on the reflection 100 , where the nuclear contribution to the neutron cross section vanishes. In Figure 1 it can be seen that the magnetic intensity disappears at the Néel temperature $T_{\mathrm{N}}=124 \mathrm{~K}$. For the strongest nuclear reflection 022 , where a magnetic contribution is negligible, we also observed a subtle intensity anomaly at $T_{\mathrm{N}}$, indicating that the onset of magnetic order induces a magnetostrictive distortion of the crystal structure. A bond-length distortion of the $\mathrm{VO}_{6}$-octahedra and hence with a strong modification of the intensity of the main nuclear Bragg reflections. This transition can be attributed to the onset of orbital order.

Our data thus imply that the orbital ordering transition occurs at a higher temperature than the magnetic transition. This sequence of transitions agrees with the conventional Goodenough- much more pronounced anomaly is observed at the higher temperature $T_{\mathrm{S}}=$ $136 \mathrm{~K}$, where magnetic order is no longer present. We will show in Section 3.2 below that this anomaly is due to a 
transition between orthorhombic and monoclinic crystal structures, which is associated with a strong Kanamori picture, according to which the exchange interactions between magnetic moments driving the magnetic transition are determined by the arrangement of orbitals. The orbital ordering pattern, in turn, is established by a cooperative Jahn-Teller distortion at a higher temperature. The sequence of transitions we find also agrees with reference [12], but the transition temperatures given there are about $20 \mathrm{~K}$ higher than the ones we observe. Our transition temperatures are in good agreement with those given in references [4] and [13], but the sequence of transitions reported there is opposite to the ones we find. In our study we have measured the $T$-dependence of the intensities of nuclear and magnetic Bragg reflections by singlecrystal neutron diffraction. This method is more sensitive than neutron powder diffraction used in reference [13], especially in the region, where the magnetic order sets in. Thus, we clearly evidenced that the magnetic transition sets in at $124 \mathrm{~K}$ and not at $136 \mathrm{~K}$ as given in reference [13].

\subsection{Crystal structure}

The crystal structure of $\mathrm{CeVO}_{3}$ was inferred from synchrotron $\mathrm{X}$-ray powder diffraction data recorded for scattering angles $5^{\circ}<2 \theta<150^{\circ}$ at $10 \mathrm{~K}$ and $160 \mathrm{~K}$, that is, well below and above the structural phase transition temperature $T_{\mathrm{S}}=136 \mathrm{~K}$. Refinements of the diffraction pattern at $160 \mathrm{~K}$ were performed in the orthorhombic space group Pbnm (standard setting Pnma), using the atomic parameters of $\mathrm{LaVO}_{3}$ (at $150 \mathrm{~K}$ ) as starting values [11]. The vanadium atoms are located at the special Wyckoff position $4 b$, and the coordinate values are consequently fixed. Seven positional parameters of the Ce-, O1- (both in $4 c$ ), and O2-atoms (in $8 d$ ) are variable. With a total number of 640 Bragg peaks the refinements resulted in a satisfactory residual $R_{F}=0.020$. The results of the refinements are summarized in Table 1 . It can be seen that the positional parameters of the oxygen atoms do not reach the precision of those of the cerium atoms, due to the smaller scattering power of the oxygen atoms in X-ray diffraction. Nonetheless, the data show that the O1- and O2- positions deviate significantly from the ideal sites $(0,1 / 2,1 / 4)$ and $(3 / 4,1 / 4,0)$ of the undistorted cubic structure. This is a consequence of relative tilting of the $\mathrm{VO}_{6}$-octahedra due to steric constraints ("ionic-size effect"). This effect also leads to a subtle distortion of the $\mathrm{VO}_{6}$-octahedra, but at $160 \mathrm{~K}$ the six $\mathrm{V}$-O-bond lengths are almost equal (deviation $<0.5 \%$ ).

Figure 2 shows that a monoclinic distortion is clearly manifested at $10 \mathrm{~K}$ by a splitting of the reflections at $2 \theta=12.70^{\circ}$. In our setting of the orthorhombic cell (Pbnm) the reflection at this angle can be indexed as 022 . Group-subgroup relations between the space groups can be generally used to find derivative structures of the perovskite type. For the orthorhombic space group $P b n m$ the monoclinic space groups $P 2_{1} / b\left(P 2_{1} / b 11\right), P 2_{1} / n(P 1$ $\left.2_{1} / n 1\right)$ and $P 2_{1} / m\left(P 1122_{1} / m\right)$ are deduced from this analysis. In the setting Pnma, as used in reference [13],
Table 1. Results of the Rietveld refinements of $\mathrm{CeVO}_{3}$. For the synchrotron powder data collected at $10 \mathrm{~K}$ and $160 \mathrm{~K}$ the crystal structures were refined in the monoclinic and orthorhombic space groups $P 2_{1} / b$ and $P b n m$, respectively. Cell parameters and interatomic distances in the $\mathrm{VO}_{6}$-octahedra are listed.

\begin{tabular}{|c|c|c|}
\hline & $\begin{array}{l}\mathrm{CeVO}_{3} \\
\text { at } 10 \mathrm{~K}\end{array}$ & $\begin{array}{c}\mathrm{CeVO}_{3} \\
\text { at } 160 \mathrm{~K}\end{array}$ \\
\hline Space group & $P 2_{1} / b$ & Pbnm \\
\hline$a[\AA]$ & $5.52347(2)$ & $5.51680(2)$ \\
\hline$b[\AA]$ & $5.57933(2)$ & $5.55429(2)$ \\
\hline$c[\AA]$ & $7.71817(3)$ & $7.79260(3)$ \\
\hline$\alpha\left[^{\circ}\right]$ & $90.0798(3)$ & 90 \\
\hline$V\left[\AA^{3}\right]$ & $237.852(2)$ & $238.780(2)$ \\
\hline$x(\mathrm{Ce})$ & $0.99220(9)$ & $0.99307(9)$ \\
\hline$y(\mathrm{Ce})$ & $0.03869(6)$ & $0.03803(5)$ \\
\hline$z(\mathrm{Ce})$ & $0.24986(9)$ & 0.25 \\
\hline$x(\mathrm{O} 1)$ & $0.0779(8)$ & $0.0834(9)$ \\
\hline$y(\mathrm{O} 1)$ & $0.4857(8)$ & $0.4851(8)$ \\
\hline$z(\mathrm{O} 1)$ & $0.2509(12)$ & 0.25 \\
\hline$x(\mathrm{O} 2)$ & $0.7007(16)$ & $0.7119(6)$ \\
\hline$y(\mathrm{O} 2)$ & $0.2826(13)$ & $0.2899(6)$ \\
\hline$z(\mathrm{O} 2)$ & $0.0391(10)$ & $0.0398(4)$ \\
\hline$x(\mathrm{O} 3)$ & $0.2798(16)$ & \\
\hline$y(\mathrm{O} 3)$ & $0.7054(13)$ & \\
\hline$B_{i s}(\mathrm{Ce})$ & $0.301(5)$ & $0.413(4)$ \\
\hline$B_{i s}(\mathrm{~V})$ & $0.275(15)$ & $0.327(13)$ \\
\hline$B_{i s}(\mathrm{O} 1)$ & $0.63(9)$ & $0.88(10)$ \\
\hline$B_{\text {is }}(\mathrm{O} 2)$ & $0.40(7)$ & $0.41(6)$ \\
\hline$B_{i s}(\mathrm{O} 3)$ & 0.40 & \\
\hline$d_{\mathrm{V} 1-\mathrm{O} 11}[\AA]$ & $1.986(9)$ & $2.0034(12)$ \\
\hline$d_{\mathrm{V} 1-\mathrm{O} 21}[\AA]$ & $1.951(8)$ & $1.996(3)$ \\
\hline$d_{\mathrm{V} 1-\mathrm{O} 22}[\AA]$ & $2.072(8)$ & $2.014(3)$ \\
\hline$d_{\mathrm{V} 2-\mathrm{O} 12}[\AA]$ & $1.972(9)$ & \\
\hline$d_{\mathrm{V} 2-\mathrm{O} 31}[\AA]$ & $1.948(8)$ & \\
\hline$d_{\mathrm{V} 2-\mathrm{O} 32}[\AA]$ & $2.069(8)$ & \\
\hline
\end{tabular}

the deduced monoclinic space groups are $P 2_{1} / n\left(P 2_{1} / n\right.$ 1 1), $P 2_{1} / m\left(\begin{array}{lll}P & 1 & 2_{1} / m \\ 1\end{array}\right)$ and $P 2_{1} / a\left(P 112_{1} / a\right)$. The observed splitting into the reflections 022 and $0-22$ (in $P b n m$ ) indicates that the monoclinic plane is the $b c$-plane (b-glide plane of Pbnm, or $a$-glide plane of Pnma) and the monoclinic angle is $\alpha$. This stringently implies that the space group of the monoclinic phase of $\mathrm{CeVO}_{3}$ is $P 2_{1} / b$ (or $P 2_{1} / a$ as a subgroup of $P n m a$ ). The same space group was already suggested by Ren et al. [12], but the authors of reference 13 reported the space group $P 2_{1} / n$. For this space group the $a c$-plane ( $n$-glide plane) is the monoclinic plane, and the monoclinic angle is $\beta$, so that one would expect a splitting of the reflection 202 instead of the 022 , contrary to our observation. Therefore the space group $P 2_{1} / n$ given in reference [13] is incorrect. In their setting (starting from Pnma) it must be $P 2_{1} / a$, The same space group was found earlier for the low-temperature phase of $\mathrm{LaVO}_{3}$ [11] as well as for the intermediate phase of $\mathrm{YVO}_{3}$ [3]. From our 


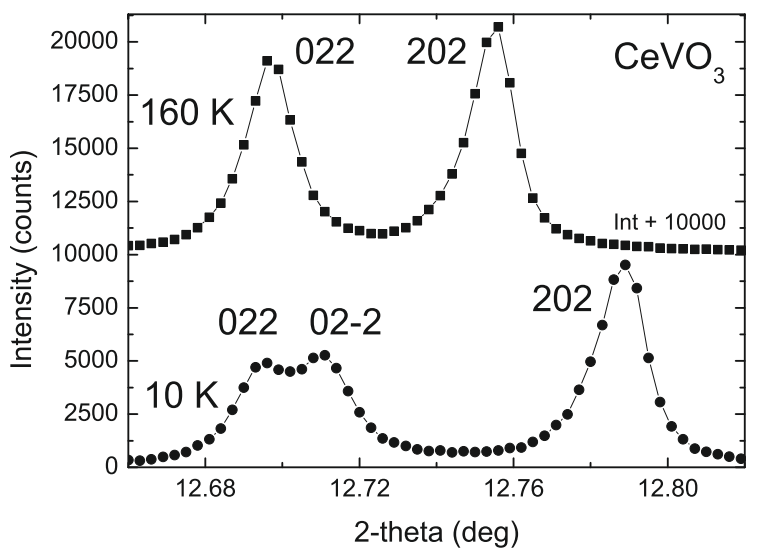

Fig. 2. Section of the synchrotron diffraction patterns at $10 \mathrm{~K}$ and $160 \mathrm{~K}$, showing the emergence of monoclinic splitting. The orthorhombic 022 reflection (space group $\mathrm{Pbnm}$ ) splits into the monoclinic reflections 022 and $02-2$ (space group $P 2_{1} / b 11$ ), whereas the peak profiles of the reflections 202 and 220 (not shown) remain unchanged.

Rietveld refinements at $10 \mathrm{~K}$ we determined a monoclinic angle $\alpha=90.0798(3)^{\circ}$. Note that the accuracy of our value exceeds that of the values $\beta=90.046(9)^{\circ}$ (at $2 \mathrm{~K}$, in $P 2_{1} / n$ ) and $\alpha=90.10^{\circ}$ (at $80 \mathrm{~K}$, in $P 2_{1} / b$ ) given in earlier works $[12,13]$.

Refinements of the diffraction pattern at $10 \mathrm{~K}$ were performed in the monoclinic space group $P 2_{1} / b$, using the atomic parameters of $\mathrm{LaVO}_{3}$ (at $10 \mathrm{~K}$ ) as starting values [11]. Here the vanadium atoms are also located in the special positions $2 c$ and $2 d$. The eight $\mathrm{O} 2$ sites $(8 d$ in $P b n m$ ) split into two 4-fold general positions (O2 and $\mathrm{O} 3$ in $4 e$ of $P 2_{1} / b$ ). The Ce- and O1-atoms are also located at the Wyckoff position $4 e$. With a total number of 1204 structure factors, all of the 12 positional parameters could be successfully refined, resulting in a residual $R_{F}=0.027$ (Tab. 1).

Our refinements also showed that the relative tilts of the $\mathrm{VO}_{6}$-octahedra in both the monoclinic and orthorhombic phases are similar. However, the V-O-bond distances are strongly modified in the monoclinic phase (Tab. 1). In particular, two of the four V-O2 bonds (almost lying in the $a b$-plane) are strongly elongated (Fig. 3 ). This supports the notion that the structural transition is driven by the cooperative Jahn-Teller effect (orbital ordering). As discussed in prior work [1-8], the stacking pattern of the octahedra is consistent with a $G$-type orbital ordering pattern built up of uniformly half-occupied $d_{x y}$ orbitals as well as $d_{x z}$ and $d_{y z}$ orbitals with alternating occupancy both in the $a b$-plane and along the $c$-axis.

Due to anomalies in the static and dynamic spin structure of the monoclinic phase of $\mathrm{YVO}_{3}$ found by neutron spectroscopy an "orbital Peierls phase" was proposed for this state [5]. This phase is characterized by a dimerization along the $z$-direction. Such a dimerization can only occur in the non-centrosymmetric structure with the space group $P b\left(\begin{array}{llll}P & b & 1 & 1\end{array}\right)$, as deduced recently from el-

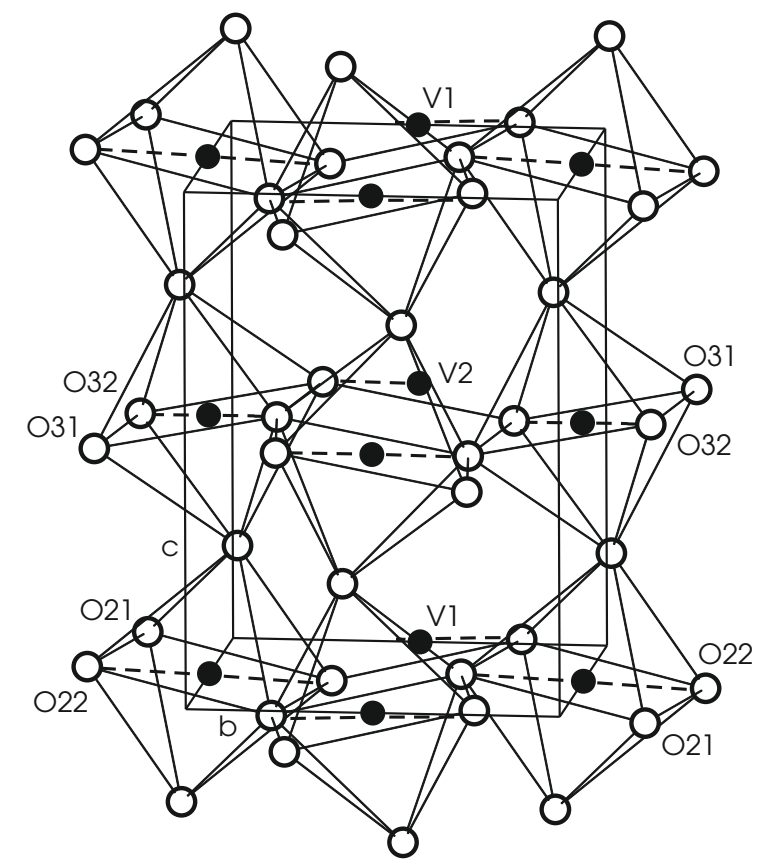

Fig. 3. Network of distorted corner-shared $\mathrm{VO}_{6}$-octahedra in the monoclinic phase of $\mathrm{CeVO}_{3}$ at $10 \mathrm{~K}$. The dashed lines represent the elongated V-O-bonds due to the Jahn-Teller effect.

lipsometric measurements [6]. $\mathrm{Pb}$ allows the possibility of dimerization along $z$, resulting in chains of alternating slightly shortened and elongated V1-V2-distances [7]. Thus a possible dimerization requires systematic shifts of the vanadium atoms from the ideal position values $z(\mathrm{~V} 1)=0$ and $z(\mathrm{~V} 2)=0.5$. This could not be detected for the vanadates by diffraction so far. From structure refinements in the space group $\mathrm{Pb}$ we obtained the parameters $z(\mathrm{~V} 1)=0.002(4)$ and $z(\mathrm{~V} 2)=0.497(3)$. The positional parameters of the vanadium atoms could not be refined with sufficient accuracy in order to see significant deviations from the ideal position values. Therefore this question could not be resolve for $\mathrm{CeVO}_{3}$.

Our observations on $\mathrm{CeVO}_{3}$ are in general agreement with other members of the series $R \mathrm{VO}_{3}$. The distortion pattern of the $\mathrm{VO}_{6}$ octahedra below $T_{\mathrm{S}}$ (four short and two long V-O bonds) agrees with the Jahn-Teller distortion pattern expected for $t_{2 g}$-orbitals occupied by two electrons in a high-spin configuration, and with observations on other pseudocubic vanadates at low temperature. Other aspects of the $\mathrm{CeVO}_{3}$ crystal structure, such as the monoclinic distortion and the octahedral tilt pattern, are also common to the entire $R \mathrm{VO}_{3}$ family. For $\mathrm{LaVO}_{3}$ and $\mathrm{YVO}_{3}$ the monoclinic angles were found to be $\alpha=90.129(6)^{\circ}$ (at $10 \mathrm{~K}$ ) and $\alpha=90.023\left(3^{\circ}\right)$ (at $80 \mathrm{~K}$ ), respectively [3,11], compared to $90.0798(3)^{\circ}$ in $\mathrm{CeVO}_{3}$. These values indicate that the monoclinic distortion is more pronounced for the vanadates containing large $R^{3+}$-ions, probably as a consequence of the ionic size effect. The unit cell volume and the octahedral tilt pattern also evolve with the size of the $R^{3+}$ ions. From La to Er the cell volume decreases continuously 
due to the well-known lanthanide contraction $[7,14]$. The lattice constants $a$ and $c$ decrease significantly, whereas $b$ increases slightly. For the compounds containing smaller $R^{3+}$-ions the $\mathrm{VO}_{6}$-octahedra are more rotated around the $b$ - and $c$-axes than along the $a$-axis of the orthorhombic structure with the space group Pbnm.

For $\mathrm{CeVO}_{3}$, a deviation from the continuous plot of the cell volumes of $R \mathrm{VO}_{3}$ compounds can be observed, and the tilt angles of the $\mathrm{VO}_{6}$-octahedra we find are more pronounced than those of adjacent members of the series $[7,14]$. A possible origin of this anomaly is a deviation of the Ce valence from the $3+$ state found in all other $\mathrm{RVO}_{3}$ compounds. Indeed, Ce is known to be tetravalent or mixed-valent in other chemical environments. A contraction of the Ce ion induced by its higher charge would explain the anomalously small unit cell volume and larger distortions found in $\mathrm{CeVO}_{3}$. This scenario is further explored below in the context of the ordered magnetic moment on the Ce sublattice.

\subsection{Magnetic structure}

The arrangement of magnetic moments on the vanadium and cerium ions was determined by single-crystal neutron diffraction experiment. From a data set collected at $150 \mathrm{~K}$, where the crystal structure is described by the orthorhombic space group Pbnm, we determined the overall scale factor and the positional parameters of cerium and oxygen atoms. Using the neutron wavelength $\lambda=2.36 \AA$ a small data set of 68 reflections (35 unique) was measured. Therefore the refinement of the positional parameters could not reach the accuracy of those obtained from the synchrotron X-ray powder experiment listed in Table 1, where a total number of 640 Bragg reflections was available. Thermal parameters were taken from the synchrotron experiment and they were not allowed to vary during the refinements. The refinement of the isotropic extinction parameter $g$ resulted in a value close to zero. This result is sensible, since the investigated crystal shows an enlarged mosaic spread, which is the same for both the orthorhombic and the monoclinic phase. The influence of the monoclinic splitting is rather small. The knowledge the overall scale factor allowed us to obtain the corrected magnetic structure factors. These were used for the refinements of the magnetic moments of the V-, and Ce-atoms with the program FullProf.

Below the Néel temperature $T_{\mathrm{N}}=124 \mathrm{~K}$ the strongest magnetic intensities were observed for the reflections 100 and 010 (in the space group $P 2_{1} / b 11$ ) indicating a $C$-type spin ordering with the spin sequence ++-- for the $\mathrm{V}$ atoms in the positions $1 / 200,1 / 201 / 2,01 / 20$ and $01 / 21 / 2$ respectively. Thus, the moments are antiferromagnetically coupled in the $a b$-plane and ferromagnetically coupled along the $c$-axis. From the intensity ratio of 100 and 010 reflections, one can infer that the moments are oriented in the $a b$-plane, forming an angle of $25^{\circ}$ with respect to the $b$-axis. The pattern of exchange interactions is compatible with the $G$-type orbital order inferred from the structural data [1-8].

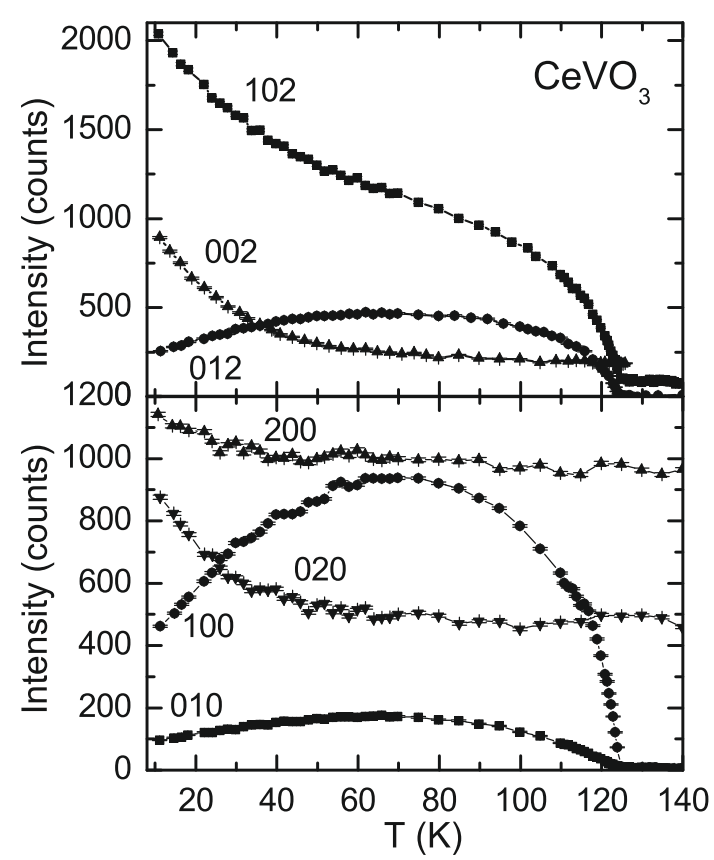

Fig. 4. Temperature dependence of magnetic Bragg reflections of $\mathrm{CeVO}_{3}$. Below 124 the C-type magnetic order of the vanadium moments appears. The decrease of the intensities 010 , 100 , and 012 , as well as the increase of that of the 102, indicates the additional induced C-type ordering of the cerium moments. Further, the intensity increase of the reflections 200, 020 , and 002 indicates the onset of an induced ferromagnetism of the cerium.

For $\mathrm{YVO}_{3}$ an additional $G$-type component was observed along the $c$-direction, whereas it is absent for $\mathrm{NdVO}_{3}$ and $\mathrm{TbVO}_{3}[5,7]$. If such a component were present, magnetic intensity would appear on the reflections 101 and 011. From the temperature dependence of the Bragg intensity we could not deduce any magnetic intensity at these positions. $G$-type ordering is therefore absent in $\mathrm{CeVO}_{3}$, as it is in other vanadates containing larger $R^{n+}$-ions [7]. Magnetic intensity is, however, observed on the reflections 200, 020 and 002. This is not observed in $R \mathrm{VO}_{3}$ with $R=\mathrm{Y}, \mathrm{Nd}$, and $\mathrm{Tb}$ and indicates a ferromagnetic component of the ordering pattern, in agreement with the macroscopic magnetic susceptibility of $\mathrm{CeVO}_{3}[2]$. Bertaut's representation analysis has shown that the basis vectors $\left(C_{x}, F_{y},-\right)$ and $\left(F_{x}, C_{y},-\right)$ are compatible with both $P 2_{1} / b$ and Pbnm [7,21]. For the Ce atoms, located in the Wyckoff position $4 c[(1) x, y$, $1 / 4$; (2) $-x,-y, 3 / 4$; (3) $1 / 2-x, 1 / 2+\mathrm{y}, 1 / 4$; (4) $1 / 2$ $+x, 1 / 2-y, 3 / 4]$ the basis vectors are defined as $\mathrm{F}(++$ $++)$ and $\mathrm{C}(++--)$. However, a combination of both modes is unexpected and indicates the presence of terms beyond the usual bilinear terms in the spin Hamiltonian, as already discussed for $\mathrm{YVO}_{3}[7]$.

In Figure 4 it can be seen that the intensities of the magnetic reflections 100, 010, 012, and 102 exhibit a nonmonotonic temperature dependence. A similar behavior has been observed for $\mathrm{NdVO}_{3}$ and $\mathrm{TbVO}_{3}$ and can be ascribed to the influence of the lanthanide moments, which 


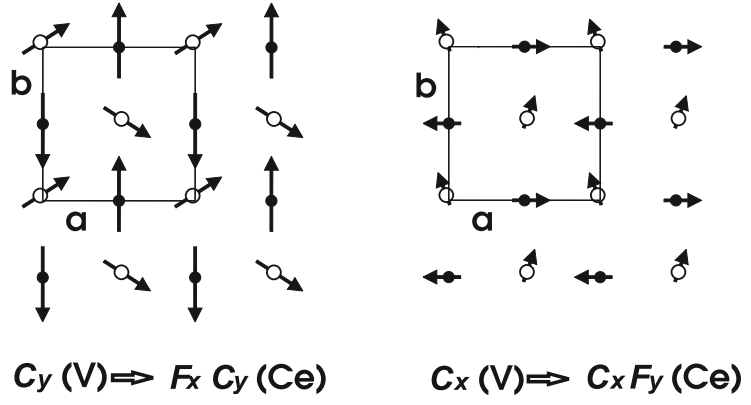

Fig. 5. Magnetic structure of $\mathrm{CeVO}_{3}$ at $10 \mathrm{~K}$. The open circles are cerium and the smaller filled circles are vanadium. The cerium atoms are at $z=1 / 7$, the vanadium atoms are at $z=0$ and $z=1 / 2$. The magnetic moments of the metal atoms are aligned in the $a b$-plane. Along the $c$-axis all the moments are coupled ferromagnetically. For clarity, the $C_{x}$-component of the cerium moment is exaggerated.

are polarized in the presence of the exchange field produced by the antiferromagnetic vanadium sublattice, resulting in a magnetic structure with the same symmetry [7]. Indeed, a refinement of the data shows that the magnetic structure on the cerium sublattice is also a superposition of $C_{x}$ and $C_{y}$ modes, and that the $\mathrm{Ce}-\mathrm{V}$ exchange interaction is ferromagnetic. The same type of coupling was observed for $\mathrm{TbVO}_{3}$, whereas the coupling between the neodymium and vanadium spins in $\mathrm{NdVO}_{3}$ was found to be antiferromagnetic [7]. The low-temperature enhancement of the ferromagnetic intensity suggests that it arises predominantly from the Ce sublattice. Indeed, a refinement of the data set at $10 \mathrm{~K}$ resulted in values of the ferromagnetic modes on the $\mathrm{V}$-sublattice close to zero. The much larger ferromagnetic component on the Ce sublattice is thus likely a consequence of ferromagnetic Ce-Ce interactions.

In order to deduce the $C$-type ordered components of the $\mathrm{V}$-, and Ce-atoms we have used for the refinements the $F_{h k l}^{2}$-values of 100, 010,102, 012, 104 and 014; for the $F$-type ordering we have used the $F_{h k l}^{2}$-values of the magnetic contribution of 200, 020, 002, 110 and 112. Taking into account a twinning in the monoclinic phase the observed reflections $h k l$ contain contributions of both $h k l$ and $h k-l$. Our data analysis showed that there is a difference between the $F^{2}$-values of only about $0.003 \%$. A sketch of the full magnetic structure resulting from our analysis is provided in Figure 5, and the temperature evolution of all of its components is presented in Figure 6. The absolute moment values at $T=10 \mathrm{~K}$ are listed in Table 2 . The saturated total moment of the vanadium ion is $\mu(\mathrm{V})=1.39(2)$ $\mu_{B}$, far below the free-ion value of $2 \mu_{B}$. The same tendency has already been found for the vanadates $\mathrm{YVO}_{3}$, $\mathrm{NdVO}_{3}$ and $\mathrm{TbVO}_{3}$ [7]. Two mechanisms have been invoked to explain the small ordered moment: an orbital contribution to the sublattice magnetization that partly offsets the spin contribution, and zero-point spin-orbital fluctuations [22-24]. In $\mathrm{CeVO}_{3}$, electrons transferred from mixed-valent $\mathrm{Ce}$ ions would also affect the ordered moment on the vanadium sublattice. However, as model cal-

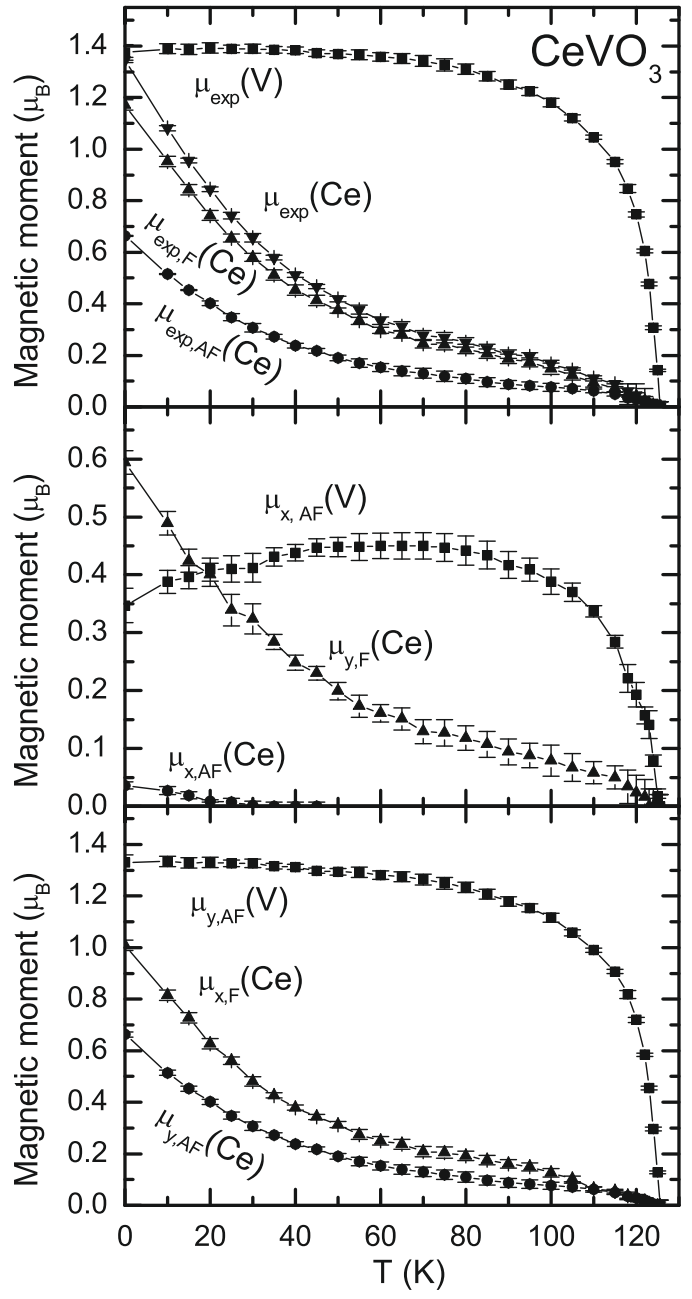

Fig. 6. Temperature dependence of magnetic moments of the cerium and vanadium atoms. Below $124 \mathrm{~K}$ the $C$-type magnetic order of the vanadium moments sets in spontaneously. The ordered vanadium sublattice induces, via Ce-V-exchange, a polarization of the cerium moments resulting in a ferrimagnetic structure.

culations aimed at separating these different contributions are still at a stage of rapid development, the ordered moment on the vanadium sublattice does not provide direct information about a possible anomalous ionic valence state in $\mathrm{CeVO}_{3}$.

The localized moment in the $4 f$-orbitals of cerium offers a more direct perspective on the ionic valence states. The total magnetic moment on the Ce-ion, obtained by extrapolating the magnetic Bragg intensities to $T=0$, is $\mu(\mathrm{Ce})=1.35(4) \mu_{B}$. This is much smaller than $g J=$ $2.14 \mu_{B}$, the theoretical moment of the $\mathrm{Ce}^{3+}$ ion (electron configuration $4 f^{1}, J=5 / 2$ ). While the magnetic moments of many insulating $\mathrm{Ce}^{3+}$ compounds (such as $\mathrm{CeOBr},[25]$ ) are in good agreement with the free-ion value, significant deviations have been observed in some oxides (such as $\mathrm{Ce}^{3+}$ impurities in ( $\left.\left.\mathrm{Sr}, \mathrm{Ca}, \mathrm{Ba}\right) \mathrm{O}[26]\right)$. Possible origins include partial quenching of the orbital moment by the crystal field, covalency with ligand ions, and orbit-lattice 
Table 2. Magnetic moments of the $\mathrm{V}$ - and Ce-atoms in $\mathrm{CeVO}_{3}$ at $10 \mathrm{~K}$ as obtained from the refinements using our single-crystal neutron diffraction data.

\begin{tabular}{lccccc}
\hline $\mathrm{Ce}$ & $\mu_{x}\left[\mu_{B}\right]$ & $\mu_{y}\left[\mu_{B}\right]$ & $\mathrm{V}$ & $\mu_{x}\left[\mu_{B}\right]$ & $\mu_{y}\left[\mu_{B}\right]$ \\
\hline$x, y, 1 / 4$ & $-0.04(1)$ & $+0.49(2)$ & $1 / 2,0,0$ & $+0.39(2)$ & \\
$-x,-y, 3 / 4$ & $-0.04(1)$ & $+0.49(2)$ & $1 / 2,0,1 / 2$ & $+0.39(2)$ & \\
$1 / 2-x, 1 / 2+y, 1 / 4$ & $+0.04(1)$ & $+0.49(2)$ & $0,1 / 2,0$ & $-0.39(2)$ & \\
$1 / 2+x, 1 / 2-y, 3 / 4$ & $+0.04(1)$ & $+0.49(2)$ & $0,1 / 2,1 / 2$ & $-0.39(2)$ & \\
\hline$x, y, 1 / 4$ & $+0.82(2)$ & $+0.51(2)$ & $1 / 2,0,0$ & & $+1.34(2)$ \\
$-x,-y, 3 / 4$ & $+0.82(2)$ & $+0.51(2)$ & $1 / 2,0,1 / 2$ & & $+1.34(2)$ \\
$1 / 2-x, 1 / 2+y, 1 / 4$ & $+0.82(2)$ & $-0.51(2)$ & $0,1 / 2,0$ & & $-1.34(2)$ \\
$1 / 2+x, 1 / 2-y, 3 / 4$ & $+0.82(2)$ & $-0.51(2)$ & $0,1 / 2,1 / 2$ & & $-1.34(2)$ \\
\hline
\end{tabular}

interactions. We note, however, that the observed moments of $R^{3+}$-ions in other members of the $R \mathrm{VO}_{3}$ family are close to the free-ion values [7]. In view of the anomalous structural properties and phase transition temperatures of $\mathrm{CeVO}_{3}$, this suggests that an admixture of the diamagnetic $\mathrm{Ce}^{4+}$-valence state into the ground state is at least partially responsible for the moment reduction.

Our results are in good overall agreement with the early work of Zubkov et al. [15], but they disagree strongly with those of Muñoz et al. [13] who reported a magnetic structure with the basis vector $\left(G_{x},-,-\right)$. This disagreement appears to be rooted in the analysis performed by Muñoz et al., rather than in the raw data they obtained. For instance, the data set shown in reference [13] also indicates magnetic intensity on the reflections 100 and/or 010, as observed in our experiment. Using the notation of Bertaut [21] the presence of these reflections clearly suggests a $C$-type ordering. In the case of a $G$-type ordering magnetic intensity is expected to appear on the positions of the reflections 101 and 011, but not on the 100 and 010 .

\section{Conclusions}

The crystal structure and the magnetic structure of $\mathrm{CeVO}_{3}$ were determined by a combination of highresolution X-ray powder diffraction and single-crystal neutron diffraction. This compound plays a special role in the series $R \mathrm{VO}_{3}$, because its unit cell volume and the critical temperatures for structural and magnetic phase transitions deviate from the smooth dependence on the ionic radii of the $R^{3+}$-ions that is characteristic for other members of the series. Owing to the high momentum-space resolution in the X-ray powder diffraction experiment, the lattice symmetries of the structural phases above and below the structural phase transition temperature $T_{\mathrm{S}}$ could be accurately determined. Below $T_{\mathrm{S}} \mathrm{CeVO}_{3}$ crystallizes in the monoclinic space group $P 2_{1} / b$ and not in $P 2_{1} / n$ as given earlier in reference [13]. The deformation of the $\mathrm{VO}_{6}{ }^{-}$ octahedra in the low-temperature phase is consistent with a Jahn-Teller distortion that lifts the orbital degeneracy of the $\mathrm{V}^{3+}$ ion. The onset of magnetic order is observed at $T_{\mathrm{N}}<T_{\mathrm{S}}$, that is, below the cooperative Jahn-Teller transition. Both the magnetic ordering pattern and the sequence of orbital and magnetic phase transitions are in accord with the standard Goodenough-Kanamori picture, but the close proximity of both transitions implies that magnetic ordering occurs in the presence of substantial orbital fluctuations. Exchange interactions between vanadium moments and paramagnetic cerium moments induce magnetic order on the Ce-sublattice, which in turn leads to a macroscopic magnetization by virtue of ferromagnetic $\mathrm{Ce}-\mathrm{Ce}$ interactions. The induced Ce-sublattice magnetization is significantly below the free-ion value. In conjunction with the anomalous structural and thermodynamic properties of $\mathrm{CeVO}_{3}$, this suggests a mixed-valent state of cerium in this compound.

We acknowledge the support of the Deutsche Forschungsgemeinschaft under grant UL 164/4.

\section{References}

1. H. Kawano, H. Yoshizawa, Y. Ueda, J. Phys. Soc. Jpn 63 , 2857 (1994)

2. H.C. Nguyen, J.B. Goodenough, J. Solid State Chem. 119, 24 (1995)

3. G.R. Blake, T.T.M. Palstra, Y. Ren, A.A. Nugroho, A.A. Menovsky, Phys. Rev. Lett. 87, 245501 (2001); G.R. Blake, T.T.M. Palstra, Y. Ren, A.A. Nugroho, A.A. Menovsky, Phys. Rev. B 65, 174112 (2002)

4. S. Miyasaka, Y. Okimoto, M. Iwama, Y. Tokura, Phys. Rev. B 68, 100406(R) (2003)

5. C. Ulrich, G. Khaliullin, J. Sirker, M. Reehuis, M. Ohl, S. Miyasaka, Y. Tokura, B. Keimer, Phys. Rev. Lett. 91, $257202(2003)$

6. A.A. Tsvetkov, F.P. Mena, P.H.M. van Loosdrecht, D. van der Marel, Y. Ren, A.A. Nugroho, A.A. Menovsky, I.S. Elfimov, G.A. Sawatzky, Phys. Rev. B 69, 075110 (2004)

7. M. Reehuis, C. Ulrich, P. Pattison, B. Ouladdiaf, M.C. Rheinstädter, M. Ohl, L.P. Regnault, S. Miyasaka, Y. Tokura, B. Keimer, Phys. Rev. B 73, 094440 (2006)

8. Y. Ren, T.T.M. Palstra, D.I. Khomskii, E. Pellegrin, A.A. Nugroho, A.A. Menovsky, G.A. Sawatzky, Nature 396, 441 (1998)

9. S. Geller, Acta Cryst. 10, 243 (1957) 
10. S. Geller, J. Chem. Phys. 24, 1236 (1956)

11. P. Bordet, C. Chaillout, M. Marezio, Q. Huang, A. Santoro, S.-W. Cheong, H. Takagi, C.S. Oglesby, B. Batlogg, J. Solid State Chem. 106, 253 (1993)

12. Y. Ren, A.A. Nugroho, A. A. Menovsky, J. Strempfer, U. Rütt, F. Iga, T. Takabatake, C.W. Kimball, Phys. Rev. B 67, 014107 (2003)

13. A. Muñoz, J.A. Alonso, M.T. Casáis, M.J. Martínez-Lope, J.L. Martínez, M.T. Fernández-Díaz, Phys. Rev. B 68, 144429 (2003)

14. M. Wollnik, Thesis, Techn. Univ. Berlin (1965)

15. V.G. Zubkov, G.V. Bazuev, G.P. Shveikin, Sov. Phys. Solid State 18, 1165 (1976)

16. S. Miyasaka, T. Okuda, Y. Tokura, Phys. Rev. Lett. 85, 5388 (2000)

17. edited by S.R. Hall, G.S.D. King, J.M. Stewart, Xtal3.4 User's Manual (University of Australia: Lamb, Perth 1995)
18. V.F. Sears, in: International Tables of Crystallography, edited by. A.J.C. Wilson (Kluwer, Dordrecht, 1992), Vol. C, p. 383

19. J. Rodriguez-Carvajal, Physica B 192, 55 (1993)

20. P. J. Brown, in: International Tables of Crystallography edited by A.J.C. Wilson (Kluwer, Dordrecht, 1992), Vol. C, p. 391

21. E.F. Bertaut, Acta Cryst. A 24, 217 (1968); see also E.F. Bertaut, J. de Phys. Coll. C1 32, 462 (1971) E.F. Bertaut, J. Magn. Magn. Mater. 24, 267 (1981)

22. P. Horsch, G. Khaliullin, A.M. Oleś, Phys. Rev. Lett. 91, 257203 (2003)

23. Z. Fang, N. Nagaosa, Phys. Rev. Lett. 93, 176404 (2004)

24. I.V. Solovyev, Phys. Rev. B 74, 054412 (2006)

25. J. Hölsä, M. Lastusaari, J. Niittykoski, R.S. Puche, Phys. Chem. Chem. Phys. 4, 3091 (2002)

26. R.W. Reynolds, Y. Chen, L.A. Boatner, M.M. Abraham, Phys. Rev. Lett. 29, 18 (1972) 